\title{
Comparaison de configurations d'essais hétérogènes en vue d'améliorer l'identification du comportement élastique orthotrope par l'erreur en relation de comportement
}

\author{
Tarek Merzouki ${ }^{1, a}$, Fodil Meraghni ${ }^{1}$, Hocine Chalal $^{1}$ et Tarak Ben ZineB ${ }^{2}$ \\ 1 Laboratoire de Physique et Mécanique des Matériaux LPMM UMR-CNRS 7554 École Nationale Supérieure d’Arts et Métiers, \\ ENSAM Metz, 4 Rue Augustin Fresnel, 57078 Metz, France \\ 2 LEMTA, Nancy-University, CNRS, 2 Rue Jean Lamour, 54519 Vandœuvre-lès-Nancy, France
}

Reçu le 21 mai 2007, accepté le 14 mai 2009

\begin{abstract}
Résumé - Cet article présente une comparaison de quatre configurations d'essais hétérogènes en vue d'optimiser l'identification des paramètres de comportement d'un matériau orthotrope élastique. La technique d'identification est basée sur la minimisation d'une norme énergétique formulée sur la base de l'erreur en relation de comportement. La norme est construite sur la base de principes variationnels. Elle exploite les champs de déformations mesurés au moyen d'une technique de mesure de champs cinématiques et les déformations calculées par le code d'éléments finis Abaqus. Le problème de minimisation est résolu itérativement par un algorithme régularisé : Levenberg-Marquardt. Une analyse de sensibilité aux bruits de mesure est réalisée afin d'illustrer la robustesse et la stabilité numérique. Deux types de bruit simulant les erreurs de mesures, ont été testés : aléatoire et systématique. Il ressort de cette étude que la configuration d'essai notée géométrie complexe donne le meilleur résultat d'identification.
\end{abstract}

Mots clés : Identification / erreur en relation de comportement / essais hétérogènes / principes variationnels / problème inverse / optimisation

\begin{abstract}
Comparison of heterogeneous configurations of tests in order to improve the identification of the orthotropic elastic behavior by the error in constitutive relation. The paper presents a comparison of four configurations of heterogeneous tests in order to optimize the identifiability of orthotropic material parameters of an elastic behavior. The technique of identification is based on minimization of an energy norm formulated on the basis of error in constitutive relation. This norm is based on variational principle. It exploits the experimental strains measurements obtained by an experimental technique and the strains calculated by finite element code. The problem of minimization is solved by a regularized algorithm : Levenberg-Marquardt. An analysis of sensitivity to the noises is carried out in order to illustrate the robustness and numerical stability. Two types of noise simulating the errors of measurement were tested : randomly distributed and systematic. It comes out from this study that the configuration of test noted complex geometry gives the best result of identification.
\end{abstract}

Key words: Identification / error in constitutive relation / heterogeneous tests / variational principles / inverse problem / optimization

\section{Introduction}

La richesse des mesures de champs (déplacements, déformations, formes propres modales, température...) est particulièrement bien adaptée à l'identification de grandeurs et de paramètres caractérisant des structures ou des matériaux tels que les paramètres de

\footnotetext{
a Auteur pour correspondance :

Tarek.merzouki@ensam.metz.fr
}

lois de comportement complexes en mécanique. Cette thématique de recherche constitue non seulement une préoccupation académique mais également un enjeu essentiel dans l'évolution des techniques originales de mesures de champs et leur exploitation dans l'identification de lois régissant des phénomènes multiphysiques [1-5]. Les méthodes d'identification inverse exploitant la richesse des mesures de champs cinématiques amènent le mécanicien à concevoir de nouvelles configurations 
d'essais mécaniques. Celles-ci utilisent des éprouvettes de géométrie complexe sollicitées par des chargements « simples ». L'idée consiste à concevoir et à optimiser des montages, des géométries et des trajets de chargement afin de solliciter « l'éprouvette-structure » en générant des champs de contraintes hétérogènes dans l'espace et dans le temps. Les essais hétérogènes sont généralement non standards et sont optimisés en vue d'améliorer l'identification des paramètres régissant la loi de comportement. Il serait alors possible d'identifier des lois de comportement complexes en exploitant un nombre réduit d'essais.

Outre les difficultés inhérentes à la mise en œuvre de mesures de champs, l'exploitation quantitative de ces mesures, à travers une approche inverse, constitue un verrou scientifique important. Dans ce sens, le présent travail constitue une contribution au développement théorique de stratégies d'extraction de paramètres inconnus caractérisant le comportement de milieux élastiques orthotropes. Ces stratégies sont basées sur des approches inverses du fait de l'absence de formulations directes entre les champs cinématiques et les sollicitations appliquées. Dans le présent article, une approche inverse fondée sur une approche variationnelle est utilisée pour l'identification des paramètres du comportement orthotrope. Celleci est basée sur la minimisation d'une norme énergétique formulée par l'erreur en relation de comportement $[6,7]$. L'erreur en relation de comportement est reformulée par Bonnet et al. [6] de façon à introduire l'écart entre le champ de déformations théoriques et les déformations expérimentales obtenues par une technique de mesure de champs cinématiques. L'identification des paramètres du comportement est effectuée par la minimisation de ce problème inverse.

L'utilisation de cette méthode de résolution nécessite le calcul d'une matrice notée $\mathrm{d} X_{i} / \mathrm{d} P_{j}$ traduisant la sensibilité des variables mesurées $\left(X_{i}\right)$ aux paramètres de la loi de comportement $\left(P_{j}\right)$. Dans la littérature, il existe plusieurs méthodes de construction de cet opérateur de sensibilité. On peut citer les méthodes analytiques directes [3] ou la méthode adjointe [8]. Certains auteurs [2] proposent une technique mixte basée sur les méthodes semi-analytiques utilisant conjointement la méthode des différences finies pour la différentiation numérique et un calcul analytique. Dans le présent travail, le choix s'est porté sur une méthode semi-analytique combinant la méthode analytique directe et la méthode des différences finies pour le calcul du gradient et ainsi que pour la construction de la matrice de sensibilité. La dérivation numérique est faite par la méthode des différences finies. Cette méthode relativement stable, permet au modèle inverse d'être couplé à un code de calcul par éléments finis EF. Le code de calcul Abaqus [9], est utilisé pour résoudre le problème direct. La mise en oeuvre d'une telle approche nécessite alors le choix pertinent d'une géométrie d'éprouvette, l'utilisation d'une méthode de mesure de champs cinématiques ainsi qu'une stratégie d'identification. Dans ce travail, nous avons testé la robustesse de la technique sur quatre configurations d'essais. Les essais hétérogènes sont conçus afin de comparer l'identification des paramètres régissant la loi de comportement élastique orthotrope. L'analyse de l'identification est menée sur la base de la comparaison de la stabilité des valeurs identifiées par rapport au choix du jeu de paramètres initiaux et sur la robustesse de traiter des données expérimentales bruitées. Deux types de bruit simulant les erreurs de mesures ont été testés : un bruit blanc aléatoire et un biais uniforme.

\section{Principe de l'erreur en relation de comportement en élasticité}

Soit une structure $\Omega$, constituée d'un matériau linéairement élastique dont les équations du problème de référence sont présentées en deux groupes :

- les conditions d'admissibilité : les équations de liaison; les équations d'équilibre et les conditions aux limites, - la relation de comportement :

$$
\sigma=C: \varepsilon(u)
$$

où $\sigma$ est le tenseur de contrainte, $\varepsilon$ est le tenseur de déformation associé au vecteur de déplacement $u, C$ est le tenseur de rigidité de la structure.

La relation de comportement a en effet un statut particulier : dans la pratique, étant donné le calcul du tenseur de rigidité à partir de mesures expérimentales, elle est souvent la moins fiable des équations du problème. On définit le couple $\left(u_{C A}, \sigma_{S A}\right)$ représentant respectivement le champ de déplacement cinématiquement admissible (CA) et le champ de contrainte statiquement (SA) admissible. Ce couple est la solution exacte du problème mécanique si et seulement si, il permet de satisfaire la relation de comportement (1): $\sigma_{S A}=C: \varepsilon\left(u_{C A}\right)$, soit $u_{C A}=u_{e x}$ et $\sigma_{S A}=\sigma_{e x}$.

Dans le cas où cette égalité n'est pas satisfaite, c'està-dire $\sigma_{S A}-C: \varepsilon\left(u_{C A}\right) \neq 0$, le couple $\left(u_{C A}, \sigma_{S A}\right)$ est une solution approximative du problème mécanique. On appelle alors erreur en relation de comportement $e_{R D C}$ associée au couple admissible $\left(u_{C A}, \sigma_{S A}\right)$ [10], la quantité définie en chaque point de la structure $\Omega$ par :

$$
e_{R D C}=\sigma_{S A}-C: \varepsilon\left(u_{C A}\right)
$$

La quantité $\sigma_{S A}-C: \varepsilon\left(u_{C A}\right)$ est donc une grandeur de type contrainte. Elle est choisie pour évaluer la pertinence du couple $\left(u_{C A}, \sigma_{S A}\right)$ et permet de quantifier l'écart à la solution exacte en terme de relation de comportement. L'erreur en relation de comportement de $e_{R D C}$ présente un avantage principal : elle fait porter « l'incertitude » sur la relation de comportement considérée comme étant la moins fiable en résolvant le problème mécanique (1). Classiquement, on mesure l'erreur en relation de comportement dans un problème linéaire, en utilisant la norme en énergie sur la structure considérée. L'erreur absolue globale est définie ainsi par :

$$
e_{R D C}=\left\|\sigma_{S A}-C: \varepsilon\left(u_{C A}\right)\right\|_{\Omega}
$$


avec

$$
\|\sigma\|_{\Omega}^{2}=\int_{\Omega}\left(\sigma: C^{-1}: \sigma\right) \mathrm{d} \Omega
$$

À partir de cette erreur globale, l'erreur relative est définie par :

$$
\epsilon=\frac{\left\|\sigma_{S A}-C: \varepsilon\left(u_{C A}\right)\right\|_{\Omega}}{\left\|\sigma_{S A}+C: \varepsilon\left(u_{C A}\right)\right\|_{\Omega}}
$$

$\epsilon$ traduit ainsi une précision permettant d'estimer la pertinence de l'approximation $\left(u_{C A}, \sigma_{S A}\right)$ sur la globalité de la structure $\Omega$.

En régime élastique, l'erreur en relation de comportement est formulée par une fonction $\psi(\varepsilon(u), \sigma, C)$ définie comme la somme des énergies potentielle et complémentaire pour un couple $(\varepsilon(u), \sigma)$ :

$$
\begin{aligned}
\psi(\varepsilon(u), \sigma, C)= & \frac{1}{2} \int_{\Omega}(\varepsilon(u): C: \varepsilon(u)) \mathrm{d} \Omega \\
& +\frac{1}{2} \int_{\Omega}\left(\sigma: C^{-1}: \sigma\right) \mathrm{d} \Omega-\int_{\partial \Omega}(u \cdot \sigma \cdot n) \mathrm{d} s
\end{aligned}
$$

Une conséquence bien connue des principes variationnels de l'élasticité montre que l'on a pour un tenseur de rigidité $C$ donné [6]

$$
\min _{C, u_{C A}, \sigma_{S A}} \psi(\varepsilon(u), \sigma, C)=0
$$

En l'absence des forces de volume, et en tenant compte des conditions aux limites, la fonction $\psi(\varepsilon(u), \sigma, C)$ peut être réécrite pour des champs de contraintes $\sigma$ statiquement admissibles et des champs de déplacements $u$ cinématiquement admissibles de façon à faire apparaître l'écart en relation de comportement comme suit :

$$
\begin{aligned}
\psi\left(\varepsilon\left(u_{C A}\right), \sigma_{S A}, C\right)= & \frac{1}{2} \int_{\Omega}\left(\sigma_{S A}-C: \varepsilon\left(u_{C A}\right)\right): C^{-1}: \\
& \left(\sigma_{S A}-C: \varepsilon\left(u_{C A}\right)\right) \mathrm{d} \Omega
\end{aligned}
$$

Remarque : à partir des équations (3), (4) et (6), la fonction $\psi(\varepsilon(u), \sigma, C)$ peut être exprimée par :

$$
\psi\left(\varepsilon\left(u_{C A}\right), \sigma_{S A}, C\right)=\frac{1}{2} e_{R D C}^{2}
$$

Cet aspect met en évidence la positivité de $\psi\left(\varepsilon\left(u_{C A}\right), \sigma_{S A}, C\right)$. Cela suggère pour le problème d'identification du tenseur de rigidité $C$ de définir la fonction de l'erreur en relation de comportement $\psi\left(\varepsilon\left(u_{C A}\right), \sigma_{S A}, C\right)$ associée aux données $\left(\varepsilon\left(u_{C A}\right), \sigma_{S A}\right)$ $\mathrm{du}$ problème de la structure $\Omega$.

En dualisant les conditions d'admissibilité, il est aisé de démontrer que la forme de la fonction $\psi(\varepsilon(u), \sigma, C)$ s'exprime de deux manières équivalentes [6] traduites par les équations suivantes :

$$
\begin{array}{r}
\psi\left(\varepsilon\left(u_{C A}\right), \sigma_{S A}, C\right)=\frac{1}{2} \int_{\Omega}\left(\sigma_{S A}-\sigma_{C A}\right): C^{-1}: \\
\left(\sigma_{S A}-\sigma_{C A}\right) \mathrm{d} \Omega \\
\psi\left(\varepsilon\left(u_{C A}\right), \sigma_{S A}, C\right)=\frac{1}{2} \int_{\Omega}\left(\varepsilon\left(u_{S A}\right)-\varepsilon\left(u_{C A}\right)\right): C: \\
\left(\varepsilon\left(u_{S A}\right)-\varepsilon\left(u_{C A}\right)\right) \mathrm{d} \Omega
\end{array}
$$

\section{Identification par minimisation de l'erreur en relation de comportement}

Dans le présent article, la procédure d'identification basée sur l'erreur en relation de comportement est appliquée pour identifier un comportement élastique orthotrope en contrainte plane. Dans le repère d'orthotropie, la loi de comportement est donnée par :

$$
\left(\begin{array}{c}
\sigma_{x} \\
\sigma_{y} \\
\sigma_{s}
\end{array}\right)=\left(\begin{array}{ccc}
Q_{x x} & Q_{x y} & 0 \\
Q_{x y} & Q_{y y} & 0 \\
0 & 0 & Q_{s s}
\end{array}\right)\left(\begin{array}{l}
\varepsilon_{x} \\
\varepsilon_{y} \\
\varepsilon_{s}
\end{array}\right)
$$

L'objectif est d'identifier les quatre paramètres de rigidité du matériau : $Q_{x x}, Q_{y y}, Q_{x y}$ et $Q_{s s}$ par analyse inverse à partir de mesures de champs de déformation $\varepsilon^{\exp }$. Ces champs de déformation sont mesurés par une technique optique à la surface de l'échantillon à tester. L'approche inverse est souvent considérée comme une procédure d'optimisation visant à réduire un écart quadratique entre les valeurs expérimentales et les grandeurs calculées. Cette approche nécessite de connaître en chaque point de la zone étudiée et à chaque incrément de chargement les champs de déformation mesurés $\left(\varepsilon^{e x p}\right)$ et les champs de déformation calculés par la méthode des éléments finis.

L'identification par analyse inverse revient en quelque sorte à identifier les valeurs des paramètres pilotant la loi de comportement pour lesquels les champs de déformation calculés $\left(\varepsilon^{\text {num }}\right)$ minimisent une erreur (fonction objective) qui traduit l'incompatibilité du couple $\left(\varepsilon^{e x p}, \varepsilon^{n u m}\right)$. Cette fonction objective est fondée sur une approche variationnelle basée sur une norme énergétique de l'erreur en relation de comportement, équation (11). En dualisant les conditions de Neumann et Dirichlet la fonction objective est définie comme suit :

$$
\psi(C)=\frac{1}{2} \int_{s}\left(\varepsilon^{n u m}(C)-\varepsilon^{e x p}\right)^{T} C\left(\varepsilon^{n u m}(C)-\varepsilon^{e x p}\right) \mathrm{d} s
$$

Où :

$-\varepsilon^{\exp }=\left(\begin{array}{l}\varepsilon_{x} \\ \varepsilon_{y} \\ \varepsilon_{s}\end{array}\right)^{\exp }$ est le champ de déformation mesuré expérimentalement;

$-\varepsilon^{n u m}=\left(\begin{array}{l}\varepsilon_{x} \\ \varepsilon_{y} \\ \varepsilon_{s}\end{array}\right)^{\text {num }}$ est le champ de déformation calculé par la méthode des éléments finis pour un tenseur de rigidité donné;

$-C=\left(\begin{array}{ccc}Q_{x x} & Q_{x y} & 0 \\ Q_{x y} & Q_{y y} & 0 \\ 0 & 0 & Q_{s s}\end{array}\right)$ est la matrice de rigidité à identifier ;

- s est la surface dans laquelle l'erreur en relation de comportement est définie.

Les paramètres définissant la loi de comportement peuvent alors être identifiés via la minimisation de la fonction de l'erreur en relation de comportement $\psi(C)$, équation (13) [7]. 
Le problème d'identification est défini comme suit :

Il faut trouver le tenseur de rigidité $C$ à partir des champs de déformation mesurés, $\varepsilon^{e x p}$, et des champs de déformation calculés, $\varepsilon^{\text {num }}$, tel que :

$$
\left\{\begin{array}{l}
C^{o p t}=\arg \min \psi(C) \\
\psi(C)=\frac{1}{2} \int_{s}\left\|L\left(\varepsilon^{n u m}(C)-\varepsilon^{\text {exp }}\right)\right\|^{2} \mathrm{~d} s \\
C=L^{T} L
\end{array}\right.
$$

La décomposition de $C$ en un produit $L^{T} L$ (décomposition de Cholesky) dans l'équation (14) permet d'améliorer le conditionnement du problème (la matrice hessienne).

La matrice $L$ s'écrit de la façon suivante :

$$
L=\left(\begin{array}{ccc}
L_{11} & L_{12} & L_{13} \\
0 & L_{22} & L_{23} \\
0 & 0 & L_{33}
\end{array}\right)
$$

Les différents termes sont donnés en fonction des paramètres à identifier :

$$
\left\{\begin{array}{l}
L_{11}=\sqrt{Q_{x x}} \\
L_{22}=\sqrt{Q_{y y}-\frac{Q_{x y}^{2}}{Q_{x x}}} \\
L_{33}=\sqrt{Q_{s s}} \\
L_{12}=\frac{Q_{x y}}{\sqrt{Q_{x x}}} \\
L_{13}=0 \\
L_{23}=0
\end{array}\right.
$$

\section{Résolution du problème inverse}

Pour résoudre le problème d'optimisation, équation (14), une méthode itérative de type gradient régularisée selon le schéma de résolution de LevenbergMarquardt $[11,12]$ est utilisée pour déterminer les paramètres $Q_{i j}$. Cette méthode d'approximation successive nécessite de calculer la direction et l'amplitude de descente en utilisant la valeur du gradient $(G)$ et la matrice hessienne $(H)$. L'évaluation de ces grandeurs est obtenue par un schéma semi-analytique. Il suffit alors de déterminer les dérivées premières qui définissent la matrice de sensibilité $M_{i j}$ à chaque itération :

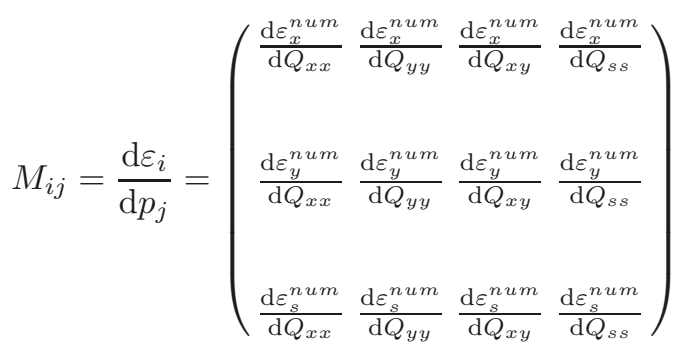

Où : $(P)$ est un vecteur dont les composantes sont les paramètres inconnus du tenseur de la matrice de rigidité à identifier $Q_{i j}$.

Le choix de la méthode d'approximation se fait en fonction du type du modèle de calcul, du degré de complexité de la loi de comportement, du nombre de paramètres à identifier et de l'existence de plusieurs minima locaux en présence de paliers. Pour un comportement non-linéaire il est préférable d'utiliser les méthodes itératives de type gradient [3].

On définit une fonction $f_{i}$ de la façon suivante :

$$
f_{i}=\left(\begin{array}{c}
f_{1} \\
f_{2} \\
f_{3}
\end{array}\right)=\left(\begin{array}{ccc}
\sqrt{Q_{x x}} & \frac{Q_{x y}}{\sqrt{Q_{x x}}} & 0 \\
0 & \sqrt{Q_{y y}-\frac{Q_{x y}^{2}}{Q_{x x}}} & 0 \\
0 & 0 & \sqrt{Q_{s s}}
\end{array}\right)
$$

$$
\times\left(\begin{array}{c}
\varepsilon_{x}^{\text {num }}-\varepsilon_{x}^{\exp } \\
\varepsilon_{y}^{n u m}-\varepsilon_{y}^{e x p} \\
\varepsilon_{s}^{\text {num }}-\varepsilon_{s}^{\exp }
\end{array}\right)
$$

La fonction $\Psi(C)$ dans l'équation (14) est écrite de la façon suivante :

$$
\psi(C)=\frac{1}{2} \int_{s}\left(f^{T} f\right) \mathrm{d} s
$$

On définit la matrice jacobienne $J_{i j}$ comme suit :

$$
\begin{aligned}
& J_{i j}=\frac{\mathrm{d} f_{i}}{\mathrm{~d} P_{j}}=\left(\begin{array}{c}
\frac{\mathrm{d} f_{1}}{\mathrm{~d} Q_{x x}} \frac{\mathrm{d} f_{1}}{\mathrm{~d} Q_{y y}} \frac{\mathrm{d} f_{1}}{\mathrm{~d} Q_{x y}} \frac{\mathrm{d} f_{1}}{\mathrm{~d} Q_{s s}} \\
\frac{\mathrm{d} f_{2}}{\mathrm{~d} Q_{x x}} \frac{\mathrm{d} f_{2}}{\mathrm{~d} Q_{y y}} \frac{\mathrm{d} f_{2}}{\mathrm{~d} Q_{x y}} \frac{\mathrm{d} f_{2}}{\mathrm{~d} Q_{s s}} \\
\frac{\mathrm{d} f_{3}}{\mathrm{~d} Q_{x x}} \frac{\mathrm{d} f_{3}}{\mathrm{~d} Q_{y y}} \frac{\mathrm{d} f_{3}}{\mathrm{~d} Q_{x y}} \frac{\mathrm{d} f_{3}}{\mathrm{~d} Q_{s s}}
\end{array}\right) \\
& =\left(L_{1}\left|L_{2}\right| L_{3} \mid L_{4}\right)\left(\begin{array}{cccc}
\triangle \varepsilon & 0 & 0 & 0 \\
0 & \Delta \varepsilon & 0 & 0 \\
0 & 0 & \Delta \varepsilon & 0 \\
0 & 0 & 0 & \Delta \varepsilon \\
& & &
\end{array}\right)+(L M)
\end{aligned}
$$


où $\left(L_{1}, L_{2}, L_{3}\right.$ et $\left.L_{4}\right)$ sont des matrices définies comme suit :

$$
\begin{aligned}
& L_{1}=\left(\begin{array}{ccc}
\frac{1}{2 \sqrt{Q_{x x}}} & \frac{-\sqrt{Q_{x y}}}{2 Q_{x x} \sqrt{Q_{x x}}} & 0 \\
0 & \frac{1}{2 \sqrt{Q_{y y}-\frac{Q_{x y}^{2}}{Q_{x x}}}} & 0 \\
0 & 0 & 0
\end{array}\right) \\
& L_{2}=\left(\begin{array}{ccc}
0 & 0 & 0 \\
0 \frac{Q_{x y}^{2}}{2 Q_{x x}^{2} \sqrt{Q_{y y}-\frac{Q_{x y}^{2}}{Q_{x x}}}} & 0 \\
0 & 0 & 0
\end{array}\right) \\
& L_{3}=\left(\begin{array}{ccc}
0 & \frac{1}{\sqrt{Q_{x x}}} & 0 \\
0 \frac{-Q_{x y}}{Q_{x x} \sqrt{Q_{y y}-\frac{Q_{x y}^{2}}{Q_{x x}}}} & 0 \\
0 & 0 & 0
\end{array}\right), L_{4}=\left(\begin{array}{ccc}
0 & 0 & 0 \\
0 & 0 & 0 \\
0 & 0 & \frac{1}{2 \sqrt{Q_{s s}}}
\end{array}\right)
\end{aligned}
$$

Où :

$$
\left(\begin{array}{l}
\triangle \varepsilon_{x} \\
\triangle \varepsilon_{y} \\
\triangle \varepsilon_{s}
\end{array}\right)=\left(\begin{array}{l}
\varepsilon_{x}^{n u m}-\varepsilon_{x}^{e x p} \\
\varepsilon_{y}^{n u m}-\varepsilon_{y}^{e x p} \\
\varepsilon_{s}^{\text {num }}-\varepsilon_{s}^{\text {exp }}
\end{array}\right)
$$

Dans le cadre de ce travail, l'algorithme de minimisation du problème inverse par la méthode de LevenbergMarquardt est le suivant :

$$
\mid \begin{aligned}
& P^{0}: \text { donnés (Jeu de paramètres initiaux) } \\
& \text { Tant que }(\|\Delta P\|<\xi) \\
& G=\frac{1}{2} \int_{s}\left(J^{T} f\right) \mathrm{d} s \\
& H=\frac{1}{2} \int_{s}\left(J^{T} J\right) \mathrm{d} s \\
& \Delta P=-(H+\lambda I)^{-1} G \\
& P^{k+1}=P^{k}+\Delta P
\end{aligned}
$$

Où :

$G$ est le gradient de la fonction $\psi(C)$;

$H$ est la matrice hessienne;

$\xi$ est un seuil fixé à une faible valeur $\left(10^{-5}\right)$;

$\lambda$ est le paramètre de régularisation.

Un test de convergence est mis en place afin d'arrêter l'algorithme de recherche. Il consiste à fixer une valeur seuil $\xi$ et à vérifier si la condition $\Delta P^{T} \Delta P<\xi$ est satisfaite.

\section{Analyse de sensibilité}

La minimisation de la fonction objective, équation (13) selon l'algorithme de Levenberg-Marquardt nécessite le calcul de la matrice de sensibilité par rapport aux paramètres recherchés $M$. Cette matrice est déterminée pour le calcul du gradient $G$ et de la matrice hessienne $H$ de la fonction objective. La matrice de sensibilité $M$ est définie comme suit :

Pour une dérivée à droite $(\delta P>0)$ ou à gauche $(\delta P<0)$

$$
M_{i j}=\frac{\varepsilon_{i}\left(C+\delta P_{j} e_{j}\right)-\varepsilon_{i}(C)}{\delta P_{j}}
$$

Où $\delta P_{j}$ est une perturbation du paramètre à identifier $P_{j}$, les composantes de la matrice $M$ étant calculées de la façon suivante :

$$
\begin{aligned}
\frac{\mathrm{d} \varepsilon_{i}^{n u m}}{\mathrm{~d} Q_{x x}}= & \frac{\varepsilon_{i}^{n u m}\left(Q_{x x}+\delta Q_{x x}, Q_{y y}, Q_{x y}, Q_{s s}\right)}{\delta Q_{x x}} \\
& -\frac{\varepsilon_{i}^{n u m}\left(Q_{x x}, Q_{y y}, Q_{x y}, Q_{s s}\right)}{\delta Q_{x x}} \\
\frac{\mathrm{d} \varepsilon_{i}^{n u m}}{\mathrm{~d} Q_{y y}}= & \frac{\varepsilon_{i}^{n u m}\left(Q_{x x}, Q_{y y}+\delta Q_{y y}, Q_{x y}, Q_{s s}\right)}{\delta Q_{y y}} \\
\frac{\mathrm{d} \varepsilon_{i}^{n u m}}{\mathrm{~d} Q_{x y}}= & -\frac{\varepsilon_{i}^{n u m}\left(Q_{x x}, Q_{y y}, Q_{x y}+\delta Q_{x y}, Q_{s s}\right)}{\left.\delta Q_{x y}, Q_{x y}, Q_{s s}\right)} \\
\frac{\mathrm{d} \varepsilon_{i}^{n u m}}{\mathrm{~d} Q_{s s}}= & \frac{\varepsilon_{i}^{\text {num }}\left(Q_{x x}, Q_{y y}, Q_{x y}, Q_{s s}+\delta Q_{s s}\right)}{\delta Q_{s s}} \\
\delta Q_{x y} & -\frac{\varepsilon_{i}^{\text {num }}\left(Q_{x x}, Q_{y y}, Q_{x y}, Q_{s s}\right)}{\delta Q_{s s}}
\end{aligned}
$$

La matrice $M(3 \times 4)$, équation $(22)$ traduit la sensibilité des trois composantes de déformation $(\varepsilon)$ par rapport aux quatre paramètres inconnus $(P)$ pour chaque point de Gauss comme indiquée dans l'équation (17) . Les composantes de la matrice $M$ sont calculées à chaque itération (k) de l'algorithme par la méthode des différences finies. Du point de vue numérique, il faut choisir le niveau de perturbation de manière à ce que la précision soit la plus grande possible. Généralement, le choix de ce paramètre est donné par : $10^{-5} P_{j} \leq \delta P_{j} \leq 10^{-3} P_{j}[4,5]$. Dans ce présent travail, la valeur de la perturbation a été choisie égale à $0,1 \%$ du paramètre $\left(P^{(k)}\right)$ à l'itération $(k)$.

\section{Optimisation de la configuration d'essai : choix de la géométrie de l'éprouvette}

Dans le cadre de ce travail, des simulations numériques par éléments finis à partir d'un comportement élastique orthotrope ont été réalisées sur des éprouvettes de formes 


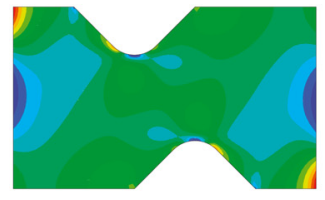

Champ de déformation mesuré par corrélation d'images

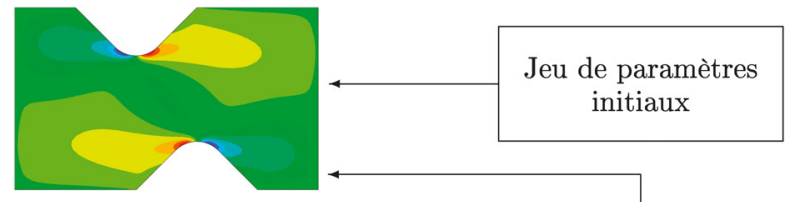

Champ de déformation simulé par le code ABAQUS

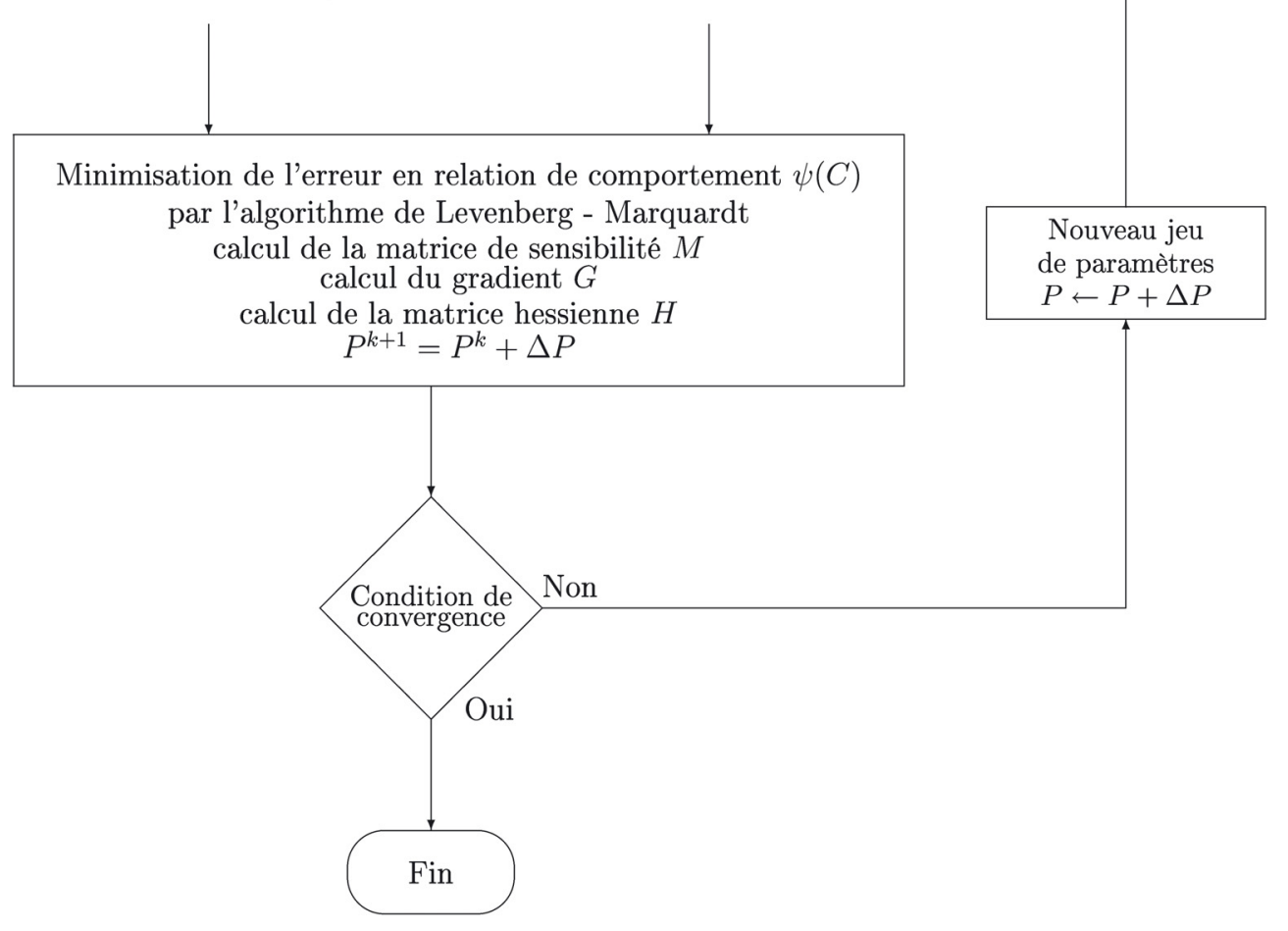

Fig. 1. Schéma synoptique de l'algorithme d'identification.

diverses. Quatre géométries ont été retenues : elles permettent d'avoir des champs de déformations suffisamment hétérogènes pour que chaque paramètre de la loi de comportement intervienne de manière sensiblement équivalente dans la réponse mécanique. La figure 2 présente les quatre formes d'éprouvettes retenues qui serviront à l'étude de l'amélioration de l'identification des paramètres de la loi de comportement étudiée. Le choix de la géométrie de ces éprouvettes est basé sur le degré d'hétérogénéité des champs de déformation et de la simplicité sur leur fabrication.

Dans la suite du travail, la procédure d'identification décrite plus haut est testée en présence de bruits de mesure qui simulent des données expérimentales. Pour une valeur de chargement identique appliquée aux quatre géométries différentes, le niveau de déformation atteint est différent d'une éprouvette à l'autre. Ainsi, le rapport signal sur bruit est également différent pour chaque configuration. Afin de mener une étude comparative dans les mêmes conditions (niveau de déformation atteint et rapport signal sur bruit), un chargement différent est appliqué à chaque configuration de manière à obtenir des niveaux de déformation moyenne équivalents pour les quatre configurations analysées avec des valeurs de paramètres initiaux identiques.

\section{Résultats de l'identification et discussion}

Dans ce travail, seulement les aspects théoriques liés à la méthode d'identification et numériques sont abordés. Dans cette partie, la procédure d'identification est menée avec des données fournies par un code de calcul éléments finis. Le modèle numérique ainsi construit permet de fournir des données expérimentales simulées, l'objectif étant ensuite de retrouver les propriétés mécaniques entrées dans le modèle numérique considérées dans la suite comme valeurs de référence. Le calcul par éléments finis a été conduit avec le code de calcul Abaqus [9]. L'essai mécanique choisi correspond à un chargement de traction uniaxial sur trois éprouvettes de géométries différentes et une traction bi-axiale sur une éprouvette cruciforme (Fig. 2). Ces configurations permettent d'obtenir un état de contrainte/déformation hétérogène pour lequel il n'existe pas une relation explicite entre le chargement appliqué et les déformations mesurées. Cette étude permettra d'optimiser la meilleure configuration parmi les quatre proposées qui aboutira à la meilleure identification des paramètres de la loi de comportement à partir d'un seul essai mécanique.

Les simulations ont été conduites, pour un matériau composite verre/époxyde, sur les géométries données par 


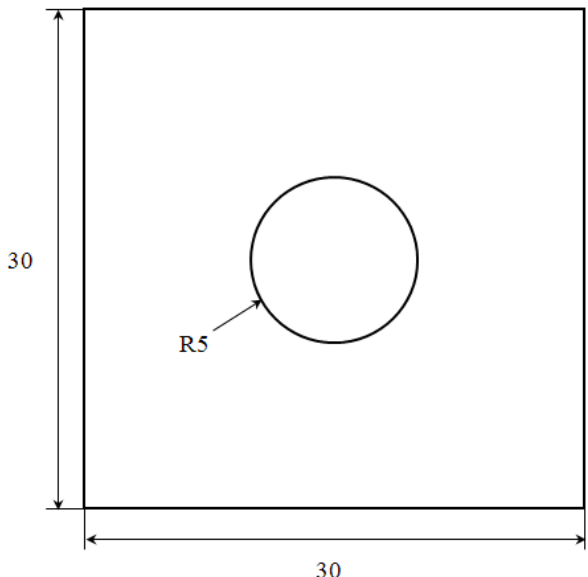

(a)

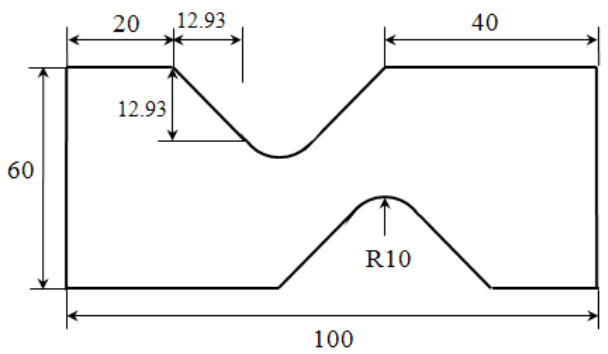

(c)

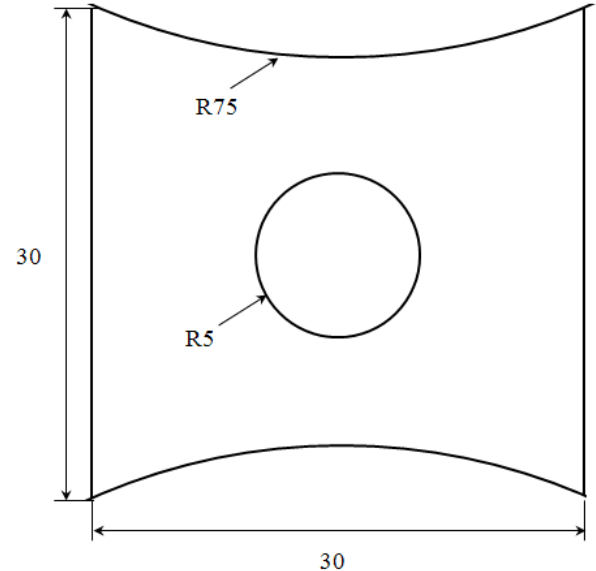

(b)

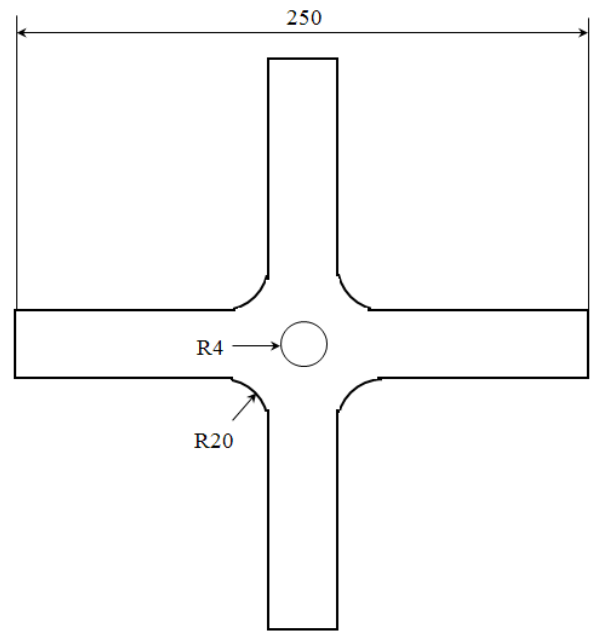

(d)

Fig. 2. Géométrie des éprouvettes utilisées lors de la procédure d'identification (dimensions en mm). (a) Plaque trouée, (b) plaque trouée à section variable, (c) éprouvette à géométrie complexe [4], (d) éprouvette cruciforme trouée.

la figure 2. L'objectif des paragraphes suivants est l'étude des aspects numériques inhérents à la sensibilité de la procédure d'identification en termes de précision et de stabilité.

\subsection{Convergence spatiale}

Dans une simulation par éléments finis, la discrétisation géométrique de l'éprouvette est un facteur essentiel contrôlant l'erreur de calcul. Une bonne estimation de la taille de l'élément fini contribuera donc à une meilleure description des gradients de déformation dans la zone étudiée. Pour cela, plusieurs simulations numériques par EF ont été menées en faisant varier la densité du maillage. Cette étude est menée seulement sur l'éprouvette rectangulaire trouée (Fig. 2). Le maillage est réalisé en utilisant des éléments finis en contrainte plane à interpolation linéaire (CSP4). Les paramètres de référence qui doivent être retrouvés par l'algorithme d'identification sont donnés dans le tableau 1.

Le résultat d'identification est donné dans le tableau 1. Avec un jeu de paramètre initial identique pour chaque simulation, les valeurs des rigidités identifiées sont exactement les mêmes que les valeurs de référence. Ce résultat montre clairement que le calcul direct s'est bien déroulé et que la procédure d'identification s'est révélée insensible au raffinement du maillage. Pour la suite du travail, un compromis entre une faible densité du maillage décrivant mal les gradients de déformation et une forte densité du maillage nécessitant un temps de calcul important est adopté. Une taille de 0,8 mm de l'élément CPS4 est choisie pour l'ensemble des configurations testées.

\subsection{Sensibilité de la procédure aux valeurs initiales}

La procédure d'identification décrite plus haut (paragraphe 3) est itérative. Elle nécessite des conditions 
Tableau 1. Influence du maillage sur les valeurs identifiées.

\begin{tabular}{|c|c|c|c|c|}
\hline & $Q_{x x}(\mathrm{GPa})$ & $Q_{y y}(\mathrm{GPa})$ & $Q_{x y}(\mathrm{GPa})$ & $Q_{s s}(\mathrm{GPa})$ \\
\hline Valeurs de référence : & 25,93 & 10,37 & 3,11 & 4,00 \\
\hline Valeurs initiales : & 50,0 & 25,0 & 1,5 & 5,3 \\
\hline \multicolumn{5}{|l|}{$\begin{array}{l}\text { Type de maillage : cas } 1 \\
\text { taille } \approx 5.6 \mathrm{~mm}\end{array}$} \\
\hline Valeurs identifiées : & 25,93 & 10,37 & 3,11 & 4,00 \\
\hline \multicolumn{5}{|l|}{$\begin{array}{l}\text { Type de maillage }: \text { cas } 2 \\
\text { taille } \approx 2.2 \mathrm{~mm}\end{array}$} \\
\hline Valeurs identifiées : & 25,93 & 10,37 & 3,11 & 4,00 \\
\hline \multicolumn{5}{|l|}{$\begin{array}{l}\text { Type de maillage : cas } 3 \\
\text { taille } \approx 0.9 \mathrm{~mm}\end{array}$} \\
\hline Valeurs identifiées : & 25,93 & 10,37 & 3,11 & 4,00 \\
\hline \multicolumn{5}{|l|}{$\begin{array}{l}\text { Type de maillage : cas } 4 \\
\text { taille } \approx 0.15 \mathrm{~mm}\end{array}$} \\
\hline Valeurs identifiées : & 25,93 & 10,37 & 3,11 & 4,00 \\
\hline
\end{tabular}

Tableau 2. Identification des propriétés élastiques : sensibilité aux valeurs initiales.

\begin{tabular}{lcccc}
\hline & $Q_{x x}(\mathrm{GPa})$ & $Q_{y y}(\mathrm{GPa})$ & $Q_{x y}(\mathrm{GPa})$ & $Q_{s s}(\mathrm{GPa})$ \\
\hline Valeurs de référence : & 25,9 & 10,3 & 3,11 & 4,00 \\
& & & & \\
Valeurs initiales : cas 1 & 50,0 & 25,0 & 1,5 & 5,3 \\
Valeurs identifiées : & 25,9 & 10,3 & 3,11 & 4,00 \\
& & & & \\
Valeurs initiales : cas 2 & 12,0 & 7,0 & 4,5 & 2,5 \\
Valeurs identifiées : & 25,9 & 10,3 & 3,11 & 4,00 \\
& & & & \\
Valeurs initiales : cas 3 & 42,0 & 5,0 & 5,8 & 1,5 \\
Valeurs identifiées : & 25,9 & 10,3 & 3,11 & 4,00 \\
\hline
\end{tabular}

initiales pour démarrer le processus d'identification. Afin d'étudier l'influence des valeurs initiales sur l'identification des paramètres, seul le résultat de l'identification pour la configuration avec une plaque rectangulaire trouée au milieu est présenté dans ce travail. Pour cette analyse, trois jeux de paramètres simulant des conditions initiales différentes ont été utilisés. Pour chaque cas, les propriétés élastiques du matériau sont identifiées. Le résultat d'identification est donné dans le tableau 2. Pour ces trois cas, les valeurs des rigidités identifiées sont exactement les mêmes et identiques aux valeurs de référence. Ces résultats montrent que l'algorithme d'identification converge vers la solution optimale minimisant l'erreur en relation de comportement, équation (13). Ceci est traduit par le fait que la fonction objective est séparément convexe et toujours définie positive.

\subsection{Sensibilité à un bruit de mesure simulé}

En pratique, la procédure d'identification serait appliquée sur des champs de déformations obtenus expérimentalement par une technique optique. Ces mesures expérimentales sont inévitablement entachées de bruits de mesure (bruit électronique, d'acquisition, intensité de lumière, déplacements hors-plan...etc). Afin 

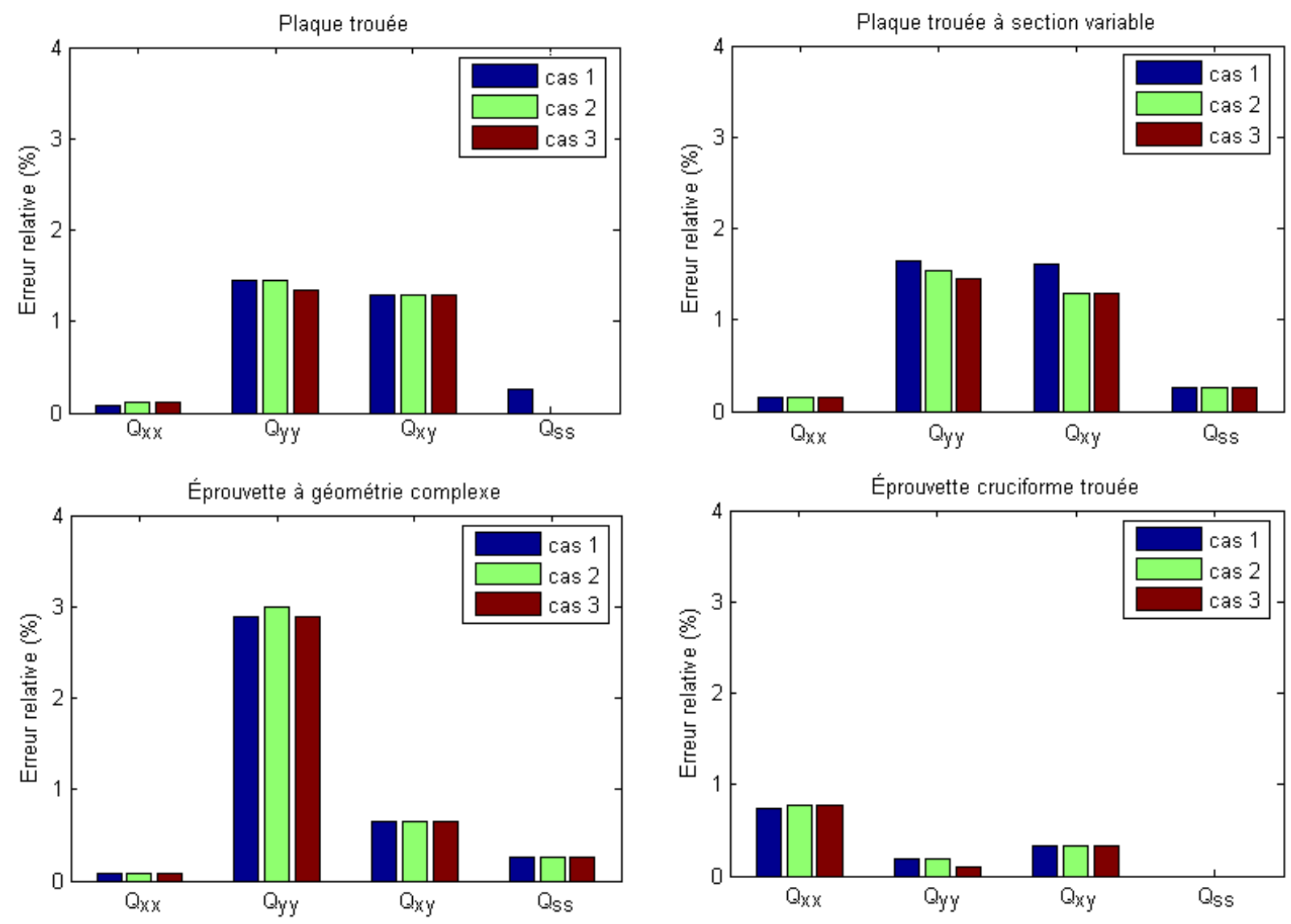

Fig. 3. Erreur relative des paramètres identifiés dans le cas d'un bruit blanc gaussien pour 3 jeux de paramètres initiaux (Tab. 2).

d'étudier la stabilité et la robustesse de la procédure d'identification en présence de données bruitées, deux types de bruits sont simulés :

1. le premier est un bruit blanc gaussien simulant une distribution aléatoire du bruit réel. Ce bruit, de moyenne nulle et d'écart type égal à $500 \mu$ déformations, est ajouté aux valeurs de déformation obtenues par le calcul EF de référence. Un traitement statistique permet de calculer la moyenne des paramètres sur 30 identifications successives conduites avec des données bruitées différentes chaque fois.

2. Le deuxième est un bruit de type biais ajouté aux valeurs de déformation obtenues par le calcul EF de référence. Il s'agit en fait d'un décalage uniforme de $500 \mu$ def sur toutes les valeurs de déformation. Ce bruit peut simuler une erreur systématique qui provoquerait des déformations parasites dues à un déplacement hors plan de l'éprouvette pendant l'essai et/ou à un mauvais positionnement de la caméra qui acquiert les champs de mesures.

La procédure d'identification tenant compte des deux types de bruit est menée sur les quatre éprouvettes (Fig. 2) et pour les trois cas de jeux de paramètres initiaux (paragraphe 7.2).

\section{Bruit blanc gaussien}

Les valeurs identifiées pour ce type de bruit sont données dans le tableau 3. La figure 3 représente l'erreur relative entre la moyenne des valeurs identifiées et celles de référence pour les quatre configurations testées. Pour chaque rigidité, l'erreur relative montre que les valeurs identifiées sont relativement indépendantes des valeurs initiales. Ce résultat montre encore une fois que l'algorithme d'identification est insensible aux valeurs initiales même en présence d'un bruit aléatoire. La figure 3 présente également une comparaison entre le résultat d'identification obtenu à partir des quatre configurations choisies. Cette comparaison montre que c'est la configuration avec une éprouvette cruciforme trouée qui présente les plus faibles écarts entre les valeurs identifiées et celles de référence sur l'ensemble des paramètres (erreur relative $<1 \%$ ). Pour cette configuration, l'identification des paramètres $Q_{y y}$ et $Q_{x y}$ a été donc améliorée par rapport aux autres configurations étudiées.

La stabilité de la procédure vis-à-vis du bruit aléatoire ajouté est représentée ici par le coefficient de variation (rapport de l'écart-type et de la valeur moyenne). Pour les quatre configurations, une grande stabilité est constatée pour l'ensemble des grandeurs identifiées et pour les différents cas de valeurs initiales (Fig. 4). 
Tableau 3. Identification avec le bruit blanc gaussien d'amplitude $=500 \mu$ def pour 3 jeux de paramètres initiaux (Tab. 2).

\begin{tabular}{|c|c|c|c|c|}
\hline & $Q_{x x}(\mathrm{GPa})$ & $Q_{y y}(\mathrm{GPa})$ & $Q_{x y}(\mathrm{GPa})$ & $Q_{s s}(\mathrm{GPa})$ \\
\hline Valeurs de référence : & 25,93 & 10,37 & 3,11 & 4,00 \\
\hline \multicolumn{5}{|l|}{ Plaque trouée } \\
\hline Valeurs initiales : cas 1 & 50,0 & 25,0 & 1,5 & 5,3 \\
\hline Valeurs identifiées & 25,91 & 10,22 & 3,07 & 3,99 \\
\hline Erreur relative $(\%)$ & 0,08 & 1,45 & 1,29 & 0,25 \\
\hline Coefficient de variation (\%) & 0,10 & 0,37 & 0,42 & 0,09 \\
\hline Valeurs initiales : cas 2 & 12,0 & 7,0 & 4,5 & 2,5 \\
\hline Valeurs identifiées & 25,90 & 10,22 & 3,07 & 4,00 \\
\hline Erreur relative $(\%)$ & 0,12 & 1,45 & 1,29 & 0,00 \\
\hline Coefficient de variation (\%) & 0,14 & 0,39 & 0,54 & 0,113 \\
\hline Valeurs initiales : cas 3 & 42,0 & 5,0 & 5,8 & 1,5 \\
\hline Valeurs identifiées & 25,90 & 10,23 & 3,07 & 4,00 \\
\hline Erreur relative $(\%)$ & 0,12 & 1,35 & 1,29 & 0,00 \\
\hline Coefficient de variation (\%) & 0,13 & 0,31 & 0,45 & 0,13 \\
\hline \multicolumn{5}{|l|}{ Plaque trouée à section variable } \\
\hline Valeurs initiale : cas 1 & 50,0 & 25,0 & 1,5 & 5,3 \\
\hline Valeurs identifiées & 25,89 & 10,20 & 3,06 & 3,99 \\
\hline Erreur relative (\%) & 0,15 & 1,64 & 1,61 & 0,25 \\
\hline Coefficient de variation $(\%)$ & 0,11 & 0,31 & 0,42 & 0,11 \\
\hline Valeurs initiales : cas 2 & 12,0 & 7,0 & 4,5 & 2,5 \\
\hline Valeurs identifiées & 25,89 & 10,21 & 3,07 & 3,99 \\
\hline Erreur relative (\%) & 0,15 & 1,54 & 1,29 & 0,25 \\
\hline Coefficient de variation (\%) & 0,11 & 0,30 & 0,39 & 0,12 \\
\hline Valeurs initiales : cas 3 & 42,0 & 5,0 & 5,8 & 1,5 \\
\hline Valeurs identifiées & 25,89 & 10,22 & 3,07 & 3,99 \\
\hline Erreur relative (\%) & 0,15 & 1,45 & 1,29 & 0,25 \\
\hline Coefficient de variation (\%) & 0,15 & 1,45 & 1,29 & 0,25 \\
\hline \multicolumn{5}{|c|}{ Éprouvette à géométrie complexe [5] } \\
\hline Valeurs initiales : cas 1 & 50,0 & 25,0 & 1,5 & 5,3 \\
\hline Valeurs identifiées & 25,91 & 10,07 & 3,13 & 3,99 \\
\hline Erreur relative (\%) & 0,08 & 2,89 & 0,64 & 0,25 \\
\hline Coefficient de variation $(\%)$ & 0,11 & 0,47 & 0,51 & 0,16 \\
\hline Valeurs initiales : cas 2 & 12,0 & 7,0 & 4,5 & 2,5 \\
\hline Valeurs identifiées & 25,91 & 10,06 & 3,13 & 3,99 \\
\hline Erreur relative (\%) & 0,08 & 2,99 & 0,64 & 0,25 \\
\hline Coefficient de variation (\%) & 0,13 & 0,49 & 0,50 & 0,10 \\
\hline Valeurs initiales : cas 3 & 42,0 & 5,0 & 5,8 & 1,5 \\
\hline Valeurs identifiées & 25,91 & 10,07 & 3,13 & 3,99 \\
\hline Erreur relative (\%) & 0,08 & 2,89 & 0,64 & 0,25 \\
\hline Coefficient de variation (\%) & 0,11 & 0,49 & 0,51 & 0,16 \\
\hline
\end{tabular}


Tableau 3. suite

\begin{tabular}{lllll}
\hline & $Q_{x x}(\mathrm{GPa})$ & $Q_{y y}(\mathrm{GPa})$ & $Q_{x y}(\mathrm{GPa})$ & $Q_{s s}(\mathrm{GPa})$ \\
\hline Valeurs de référence : & 25,93 & 10,37 & 3,11 & 4,00 \\
\hline Éprouvette cruciforme trouée & & & & \\
\hline Valeurs initiales : cas 1 & 50,0 & 25,0 & 1,5 & 5,3 \\
Valeurs identifiées & 25,74 & 10,35 & 3,12 & 4,00 \\
Erreur relative (\%) & 0,73 & 0,19 & 0,32 & 0,00 \\
Coefficient de variation (\%) & 0,34 & 0,12 & 0,83 & 0,24 \\
& & & & \\
Valeurs initiales : cas 2 & 12,0 & 7,0 & 4,5 & 2,5 \\
Valeurs identifiées & 25,73 & 10,35 & 3,12 & 4,00 \\
Erreur relative (\%) & 0,77 & 0,19 & 0,32 & 0,00 \\
Coefficient de variation (\%) & 0,32 & 0,11 & 0,82 & 0,25 \\
& & & & \\
Valeurs initiales : cas 3 & 42,0 & 5,0 & 5,8 & 1,5 \\
Valeurs identifiées & 25,73 & 10,36 & 3,12 & 4,00 \\
Erreur relative (\%) & 0,77 & 0,10 & 0,32 & 0,00 \\
Coefficient de variation (\%) & 0,33 & 0,12 & 0,82 & 0,25 \\
\hline
\end{tabular}
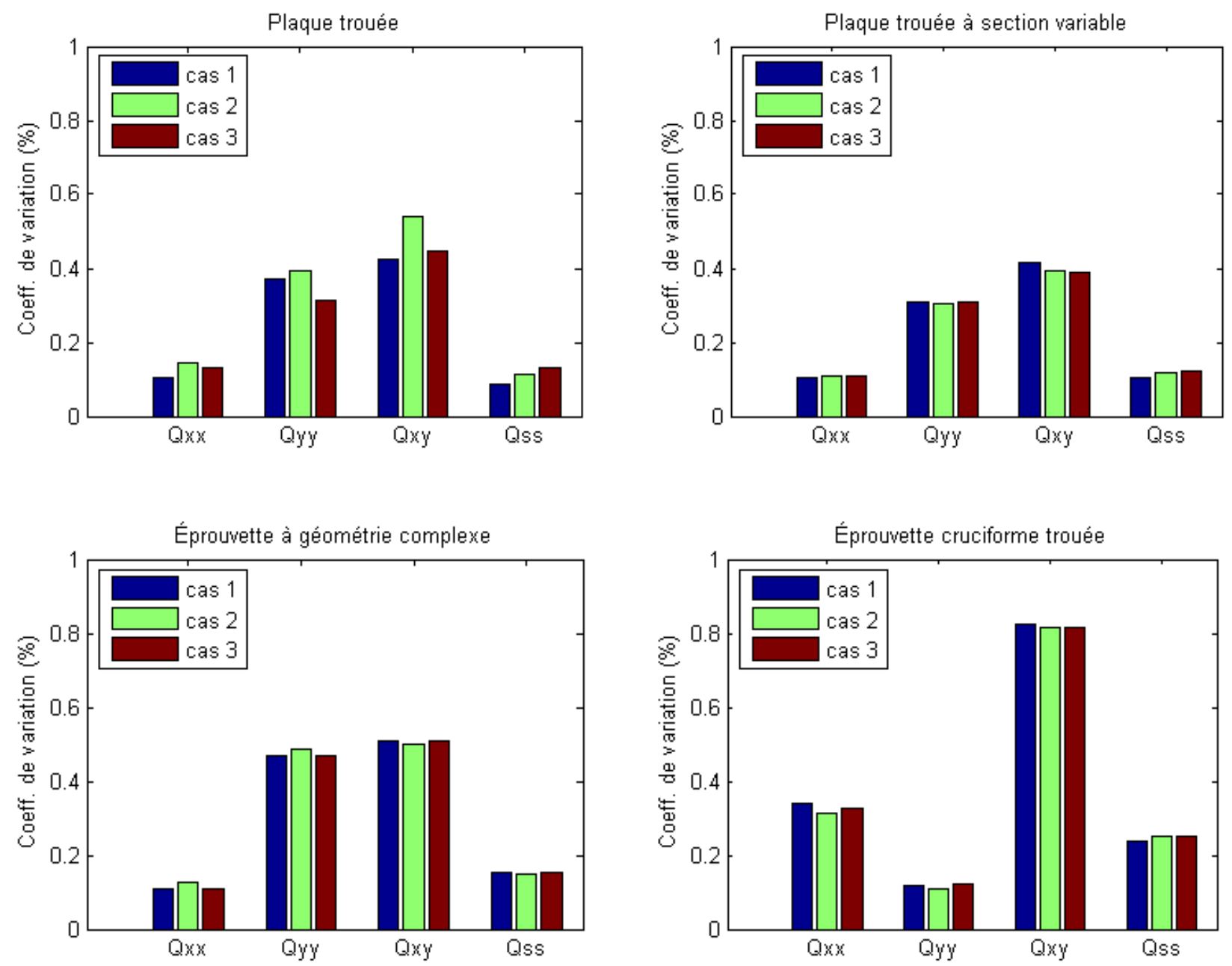

Fig. 4. Dispersion des paramètres identifiés dans le cas d'un bruit blanc gaussien. 
Tableau 4. Identification avec le bruit biais d'amplitude $=500 \mu$ def.

\begin{tabular}{lllll}
\hline & $Q_{x x}(\mathrm{GPa})$ & $Q_{y y}(\mathrm{GPa})$ & $Q_{x y}(\mathrm{GPa})$ & $Q_{s s}(\mathrm{GPa})$ \\
\hline Valeurs de référence & 25,93 & 10,37 & 3,11 & 4,00 \\
\hline
\end{tabular}

\begin{tabular}{lllll} 
Plaque trouée & & & \\
Valeurs identifiées & 24,55 & 11,12 & 2,74 & 4,01 \\
Erreur relative (\%) & 5,32 & 7,23 & 11,90 & 0,25 \\
\hline
\end{tabular}

\begin{tabular}{|c|c|c|c|c|}
\hline \multicolumn{5}{|c|}{ Plaque trouée à section variable } \\
\hline Valeurs identifiées & 24,61 & 11,08 & 2,73 & 4,01 \\
\hline Erreur relative (\%) & 5,09 & 6,85 & 12,22 & 0,25 \\
\hline \multicolumn{5}{|c|}{ Éprouvette à géométrie complexe [5] } \\
\hline Valeurs identifiées & 24,91 & 10,37 & 2,70 & 4,00 \\
\hline Erreur relative (\%) & 3,93 & 0,00 & 13,18 & 0,00 \\
\hline \multicolumn{5}{|c|}{ Éprouvette cruciforme trouée } \\
\hline Valeurs identifiées & 24,15 & 9,99 & 2,32 & 4,02 \\
\hline Erreur relative (\%) & 6,86 & 3,66 & 25,40 & 0,50 \\
\hline
\end{tabular}

\section{Bruit de type erreur de biais}

Pour ce type de bruit, le résultat de l'identification en terme d'erreurs relatives entre les paramètres identifiés et de référence est présenté dans la figure 5. L'ordre de grandeur des erreurs relatives est plus important que celui observé dans le cas d'un bruit à distribution aléatoire. En effet, l'erreur relative pour le paramètre $Q_{x y}$ est la plus importante, indiquant que ce paramètre influence peu la réponse mécanique de l'éprouvette pour les quatre configurations testées. Inversement, la rigidité de cisaillement est la plus stable, ce qui traduit la prédominance du cisaillement dans ces configurations d'essais.

\section{Conclusion}

Dans cet article, une procédure d'identification par analyse inverse a été utilisée pour déterminer les paramètres pilotant le comportement d'un matériau élastique orthotrope. La méthode d'identification a été construite sur la base d'une approche variationnelle conduisant à la minimisation d'une norme énergétique formulée par l'erreur en relation de comportement. L'algorithme d'identification développé a permis de comparer l'identification de quatre configurations d'essai mécanique hétérogènes. Celles-ci diffèrent par leurs géométries, les conditions aux limites et le chargement. L'analyse de stabilité de l'algorithme et la comparaison de l'identification ont été menées sur des champs de déformation simulés considérés comme espace de données expérimentales.
Globalement, la robustesse de la méthode d'identification proposée a été démontrée notamment au regard du choix du jeu de paramètres initiaux et en terme de stabilité de la convergence dans le cas de données expérimentales bruitées. Compte tenu de la formulation variationnelle de l'algorithme d'identification par l'erreur en relation de comportement, les résultats obtenus montrent qu'en absence de bruit de mesure, l'algorithme d'identification converge vers une solution unique. Cette stabilité est conforme à la convexité de la fonction objective traduisant l'erreur en relation de comportement.

En ce qui concerne la sensibilité de l'algorithme d'identification vis-à-vis de données bruitées, deux types de bruits ont été étudiés : un bruit blanc gaussien et un bruit biais. Dans le cas d'un bruit blanc gaussien, les valeurs moyennes identifiées sont proches des paramètres de référence et présentent des dispersions faibles. Dans le cas d'un bruit de biais, les résultats obtenus montrent l'importance de ce type de bruit dû à des erreurs systématiques telles que les déplacements hors-plan. En effet, ces déplacements introduisent des mouvements parasites interprétés par le système de mesure (caméra $\mathrm{CCD}, \ldots)$ comme des déformations. Une attention particulière doit être portée lors de validation expérimentale de la méthode d'identification (en cours) afin d'améliorer plus finement la qualité de mesure.

À partir de l'analyse de sensibilité aux valeurs initiales et aux deux types de bruits ajoutés, la configuration d'essai notée géométrie complexe [4] offre le meilleur résultat d'identification pour l'ensemble des paramètres de la loi de comportement. En effet, dans le cas d'un bruit aléatoire, cette configuration a permis d'obtenir un 


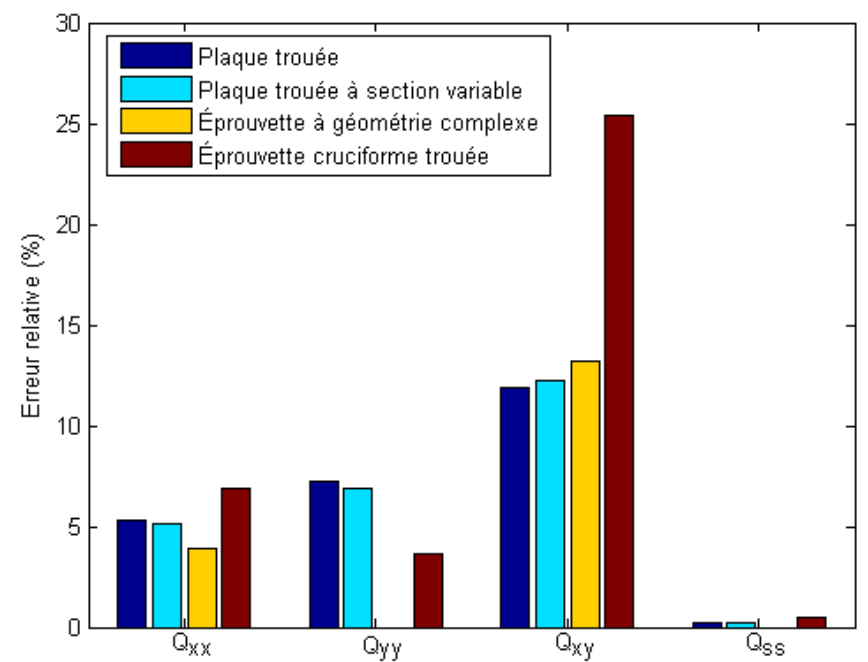

Fig. 5. Erreur relative des paramètres identifiés dans le cas d'un bruit biais.
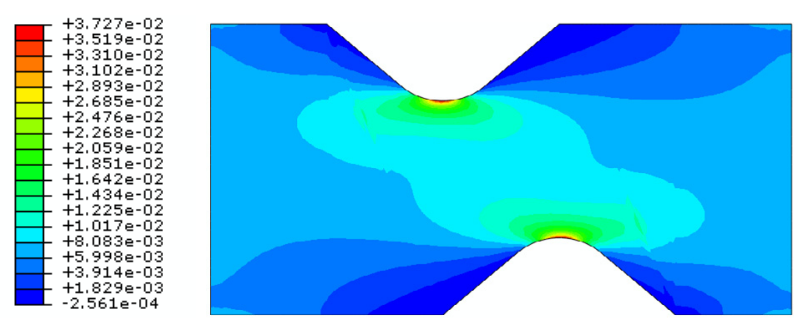

(a)

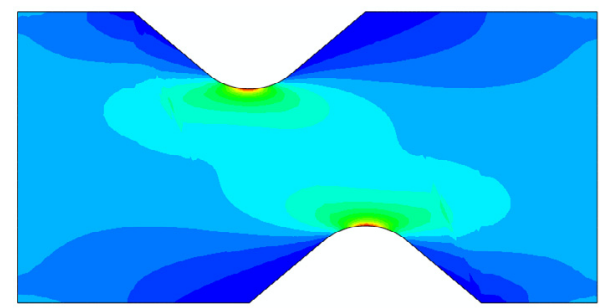

(b)

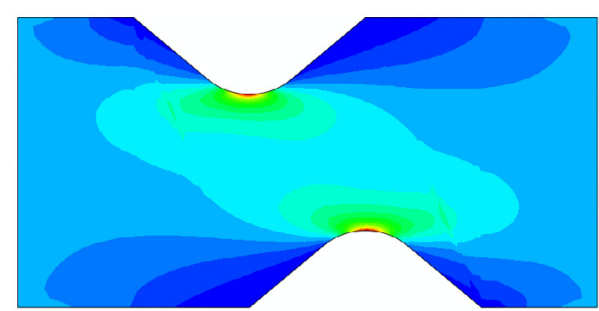

(c)

Fig. 6. Cartographies de déformation longitudinale $\varepsilon_{x}$ simulée, correspondant aux paramètres, (a) de référence, (b) identifiés dans le cas d'un bruit blanc gaussien, (c) identifiés dans le cas d'un bruit biais. La similitude des champs de déformation confirme la stabilité de de la procédure d'identification développée et son aptitude à identifier les paramètres de comportement à partir de champs de déformation contenant un bruit aléatoire ou de biais.
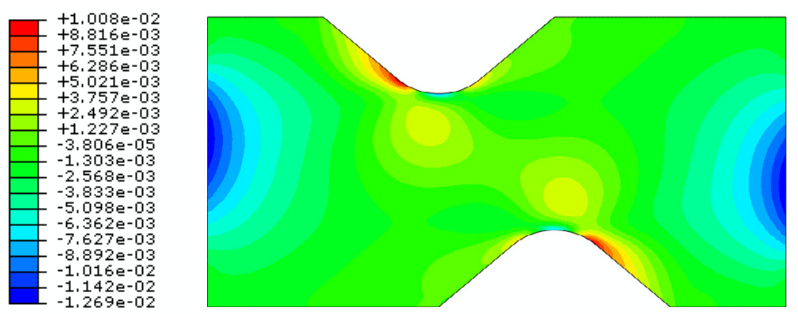

(a)

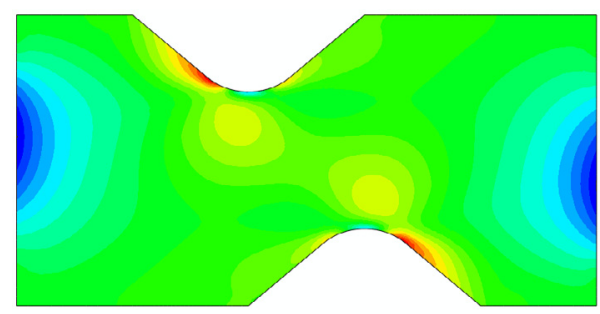

(b)

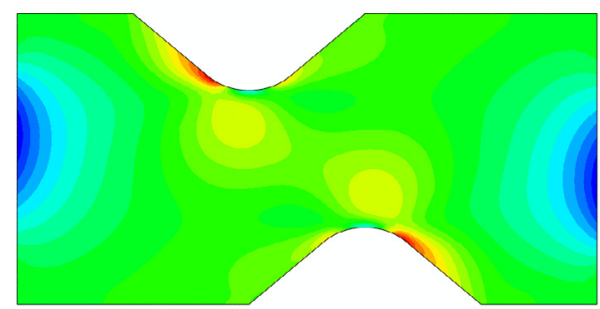

(c)

Fig. 7. Cartographies de déformation transversale $\varepsilon_{y}$ simulée, correspondant aux paramètres, (a) de référence, (b) identifiés dans le cas d'un bruit blanc gaussien, (c) identifiés dans le cas d'un bruit biais.

résultat d'identification proche de celui trouvé dans les trois autres configurations. Néanmoins, le résultat, obtenu dans le cas d'un bruit de type biais, montre que la configuration ayant la géométrie complexe est la moins sensible à ce type de bruit. Cette étude a permis de déterminer la configuration optimale pour laquelle l'identification des paramètres de la loi de comportement considérée est améliorée. Les Figures (6, 7 et 8) présentent les champs de déformations de la configuration à géométrie complexe. Ces champs correspondent aux paramètres identifiés pour les deux types de bruits ajoutés, et sont comparés aux champs obtenus à partir des paramètres de références, pour les mêmes conditions d'essai. Les champs présentés montrent la grande similitude entre les trois champs, ceci peut s'expliquer par le fait que les paramètres identifiés sont très proches des paramètres de références.

Les travaux en cours permettront d'identifier le comportement d'un matériau orthotrope à partir d'un champ de déformation mesuré par la méthode de corrélation d'images. Les développements expérimentaux tiendront 

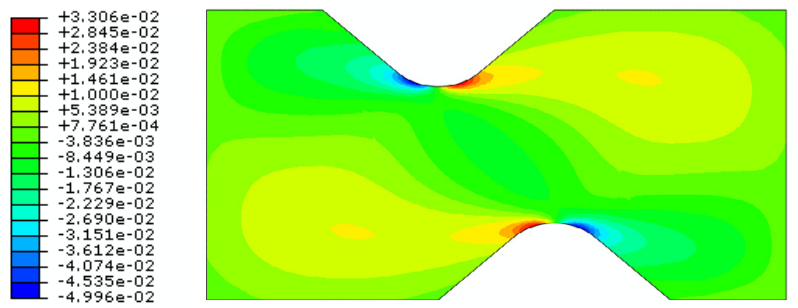

(a)

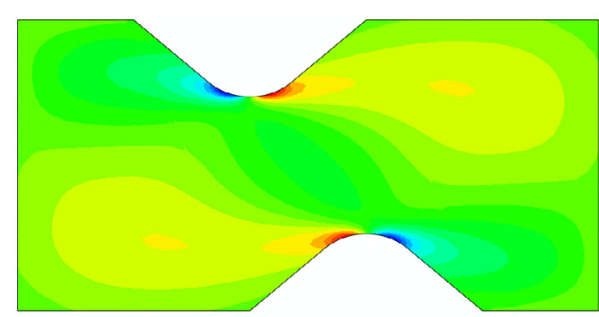

(b)

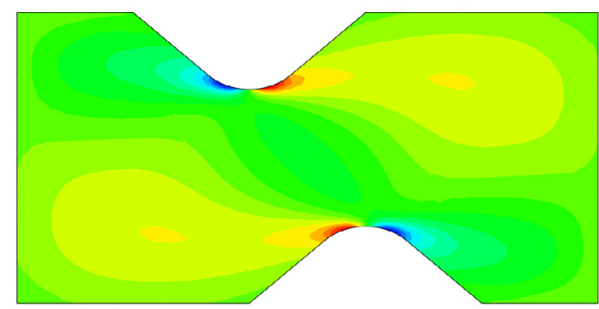

(c)

Fig. 8. Cartographies de déformation de cisaillement $\varepsilon_{s}$ simulée, correspondant aux paramètres, (a) de référence, (b) identifiés dans le cas d'un bruit blanc gaussien, (c) identifiés dans le cas d'un bruit biais.

compte des résultats obtenus par la présente étude quant au choix de la configuration pour laquelle l'identification est optimale.

\section{Références}

[1] H. Chalal, F. Meraghni, F. Pierron, M. Grédiac, Direct identification of damage behaviour of composite materials using the virtual fields method, Composites Part A 35 (2004) 841-848

[2] R. Forestier, Développement d'une méthode d'identification de paramètres par analyse inverse couplée avec un modèle éléments finis $3 \mathrm{D}$, Thèse de doctorat, ENSM de Paris, 2004

[3] A. Gavrus, E. Massoni, J.L. Chenot, The rheological parameter identification formulated as an inverse finite element problem, Inverse Problems in Engineering 7 (1999) $1-41$

[4] M.H.H. Meuwissen, C.W.J. Oomens, F.P.T. Baaijens, R. Petterson, J.D. Janssen, Determination of the elastoplastic properties of aluminium using a mixed numericalexperimental method, Journal of Materials Processing Technology 75 (1998) 204-211

[5] M.H.H. Meuwissen, An Inverse Method for the Mechanical Characterization of Metals, Thèse de doctorat, Eindhoven University of Technology, the Netherlands, 1998

[6] M. Bonnet, H.D. Bui, A. Constantinescu, Principes variationnels et exploitation de mesures de champs en élasticité, Mécanique et Industries 4 (2003) 687-697

[7] A. Constantinescu, On the identification of elastic moduli from displacement-force boundary measurements, Inverse Problems in Engineering 1 (1995) 293-315

[8] D.A. Tortorelli, P. Michaleris, Design sensitivity analysis : overview and review, Inverse Problems in Engineering 1 (1994) 71-105

[9] HKS Inc, ABAQUS Theory and Users Manuals, V. 6.6, 2006

[10] P. Ladevèze, J.P. Pelle, La maîtrise du calcul en mécanique linéaire et non linéaire, Hermès, 2001

[11] K. Levenberg, A method for the solution of certain nonlinear problems in least squares, Questions of Applied Mathematics 2 (1944) 164-168

[12] D.W. Marquardt, An algorithm for least-squares estimation of non-linear parameters, Journal on Applied mathematics 11 (1963) 431-441 